\title{
CUATRO CARTAS DIRIGIDAS A GREGORIO MARTÍNEZ SIERRA
}

\author{
Sonia NÚÑEZ PUENTE \\ Universidad Rey Juan Carlos \\ sonia.puente@urjc.es
}

Resumen: El artículo recoge la publicación de cuatro cartas inéditas de la correspondencia privada de Gregorio Martínez Sierra. Las cartas corresponden a un periodo de tiempo comprendido entre 1920 y 1943 y están dirigidas a Gregorio Martínez Sierra por Enrique Gómez Carrillo, Juan Ignacio Luca de Tena y Manuel de Falla. Asimismo, el artículo contextualiza la correspondencia de Martínez Sierra en un periodo histórico y literario concreto.

Abstract: The article makes it possible to publish for the first time four letters addressed to Gregorio Martínez Sierra. The letters published cover a period of time form 1920 until 1943. The letters are written by Enrique Gómez Carrillo, Juan Ignacio Luca de Tena and Manuel de Falla. Furthermore, the article contextualizes the correspondence to Gregorio Martínez Sierra in a historical and literary background.

Palabras clave: Correspondencia inédita. Gregorio Martínez Sierra.

Key Words: Unpublished Correspondence. Gregorio Martínez Sierra. 
Gregorio Martínez Sierra mantuvo una fructífera correspondencia con empresarios, actores, actrices y autores de su época con los que colaboró en distintos proyectos ${ }^{1}$. En este artículo hemos seleccionado cuatro cartas inéditas, dirigidas a Martínez Sierra, durante el periodo comprendido entre los años 1920 y 1943.

Estas cartas, dos de ellas manuscritas y dos mecanografiadas, no habrían podido ser publicadas sin la generosidad de Enrique Fuster del Alcázar (2006), heredero del magnífico archivo personal de Gregorio Martínez Sierra y Catalina Bárcena.

La importancia de las cartas seleccionadas radica en la información tanto personal como del ámbito literario que proporcionan y que nos facilitan la comprensión de la realidad literaria, inserta en una situación histórica y personal concreta. Se pretende, por tanto, situar la presente selección de la correspondencia de Gregorio Martínez Sierra en su contexto literario e histórico.

Siguiendo este propósito, comenzaremos el análisis de la primera de las cartas dirigida a Gregorio Martínez Sierra. Es una carta autógrafa de Enrique Gómez Carrillo, cuyo verdadero nombre era Enrique Gómez Tible. Gómez Carrillo, nacido en Guatemala en 1873, fue un destacado cronista de prosa modernista. En 1891 consiguió, tras una recomendación de Rubén Darío, una beca para estudiar en España. Antes de trasladarse a España, recaló en París, donde conoció y mantuvo relaciones con escritores como Verlaine, Leconte de Lisle y Moréas. Fue director de El Nuevo Mercurio (1907) y Cosmópolis (1919-1922).

El novelista y crítico literario guatemalteco hace referencia en su carta a la colaboración de Martínez Sierra en la revista Cosmópolis, publicación que, en 1894, era considerada una revista de vanguardia, en la que sus integrantes expresaban su deseo de tomar parte en una lucha sin tregua contra un medio donde, según ellos, estaba atrofiado el espíritu por la indiferencia. Manifestaban, de este modo, su intención de ruptura con toda expresión literaria ajena a las innovaciones. En el diálogo que constituyó la primera editorial de la revista se constata la confrontación entre la literatura nacional y, tal como se denomina en Cosmópolis, el cosmopolitismo. En este primer número intervienen, entre otros, Pedro-Emilio Coll, Pedro César Dominici y Luis Urbaneja Achelpoh (Rafael, 2001). A pesar del corto periodo de existencia de esta

1 Véase el archivo Gregorio Martínez Sierra y Catalina Bárcena, propiedad de Enrique Fuster del Alcázar. 
revista, de mayo de 1894 a julio de 1895, su circulación fue de primordial importancia. En este contexto concreto hallamos la colaboración de Martínez Sierra en Cosmópolis a la que se refiere Gómez Carrillo en su carta.

Al final de la carta, Gómez Carrillo da su autorización a Martínez Sierra para la publicación en la colección Miniatura de su obra Nuestra Señora de los Ojos Verdes. Esta obra fue publicada por primera vez en el número 144 de El Cuento Semanal, en 1909, con diez ilustraciones de Varela y en la portada aparecía una caricatura de Montagud. La obra tuvo varias ediciones más en 1913, hasta que fue publicada en la Biblioteca Estrella en la colección Miniatura, en 1919, mencionada en la carta de Gómez Carrillo.

La segunda de las cartas de nuestra breve selección es una epístola autógrafa de Juan Ignacio Luca de Tena, escrita en enero de 1938. Luca de Tena, hijo del periodista Torcuato Luca de Tena y Álvarez Ossorio, se ocupó de tareas periodísticas en la empresa familiar. Fue diputado a Cortes por Sevilla en 1929 y director de $A B C$ desde 1929 hasta 1940 . Desde $A B C$ puso en marcha una insistente campaña contra la II República, ayudado por un grupo de colaboradores tradicionalistas, liderado por Ramiro de Maeztu. Durante la Guerra Civil $A B C$ pasó a manos del gobierno republicano y Juan Ignacio Luca de Tena fundó, por su parte, el $A B C$ de Sevilla, uno de los más decididos apoyos de Franco.

En la carta de Luca de Tena se hace referencia explícita a la situación histórica de la España del momento, inmersa en plena Guerra Civil, así como a la situación personal tanto de Luca de Tena como de Gregorio Martínez Sierra, quien se encontraba en esas fechas en un estado de salud delicado. Asimismo, Luca de Tena hace mención a una obra superrealista, cuya protagonista es una sirena. La obra en cuestión es Espuma de mar, una fábula escénica en tres actos, en prosa y verso, publicada en 1940, que fue estrenada, en el mismo año, en el Teatro Lara.

La tercera carta que hemos seleccionado, dirigida a Gregorio Martínez Sierra, está escrita, de nuevo, por Juan Ignacio Luca de Tena, en julio de 1938. En ella, Luca de Tena hace mención a su obra Yo soy Brandel, segunda parte de ¿Quién soy yo? y responde a una petición de Martínez Sierra para poder estrenar la obra. Luca de Tena manifiesta su interés en conocer quién será el primer actor y recomienda a Catalina Bárcena, como primera actriz, para Espuma del mar. La carta finaliza haciendo mención a la situación de la Guerra Civil, relatando Luca de Tena a Martínez Sierra cómo el ejército del bando nacional avanzaba hacia Valencia, previendo la llegada a Madrid. 
La última carta que forma parte de nuestra selección es la que el músico Manuel de Falla escribió a Martínez Sierra en 1943. En esta carta mecanografiada Falla se queja insistentemente de la merma que sufre continuamente en la percepción de los derechos de autor. Concretamente pretende que la entidad Galaxi de NuevaYork le envíe directamente los derechos que le corresponden por sus obras.

En 1914 Gregorio Martínez Sierra y María Lejárraga conocieron, gracias a Joaquín Turina, a Manuel de Falla (O’Connor, 1987). Un año más tarde, en 1915, Martínez Sierra se convertiría en el director empresario del Teatro Eslava de Madrid, donde se presentará, en septiembre de 1916, la Compañía Cómico Dramática Gregorio Martínez Sierra con Catalina Bárcena como primera actriz.

Durante esos años se estable una estrecha colaboración entre Gregorio Martínez Sierra y Manuel de Falla, que idea su música en términos escénicos, participando en distintos proyectos con el dramaturgo (Reyero, 1980). Falla llega incluso a trasladarse a Barcelona, en compañía de María Lejárraga y el propio Martínez Sierra, para trabajar con ellos en distintos montajes teatrales (Martínez Sierra, 2000). En los casi seis meses que pasó en Barcelona finaliza las Noches en los jardines de España y ultima una nueva orquestación de El amor brujo. En 1917 llegan los «Ballets Russes» y Diaghilev, al que menciona en la carta dirigida a Martínez Sierra, y al que solicita, sin éxito, una adaptación para ballet. También se interesa Diaghilev, en este sentido, al conocer el proyecto de El Corregidor y la Molinera, en el que Falla trabajaba y del que también reclama, en la carta escrita a Martínez Sierra, los derechos de autor. El sombrero de tres picos o El Corregidor y la Molinera, basada en la obra de Pedro Antonio de Alarcón, con adaptación de Martínez Sierra, se estrenó en el Teatro Eslava de Madrid en 1917 (Santiago y Miras, 2002). Finalmente, esta obra sufrió diversas modificaciones a instancias de Sergei Diaghilev. Siguiendo las directrices de Diaghilev, Gregorio Martínez Sierra se propuso realizar las adaptaciones necesarias para introducir numerosos cuerpos de baile y Falla reformuló la obra para una gran orquesta (Checa Puerta, 1998). Esta segunda versión, con el nombre de $E l$ sombrero de tres picos se estrenó, con decorados de Picasso, en el Eslava en 1919 (Fuster del Alcázar, 2003).

Entre los libretos para ballets que escribió también Martínez Sierra, destaca, sobre todo, la escritura, entre finales de 1914 y principios de 1915, del argumento y el texto de El amor brujo, al que Falla pondría música, y que se estrenó ese mismo año, en versión escénica, en el Teatro Lara de Madrid, con 
Pastora Imperio como protagonista. Ya en 1916 se estrenó en versión sinfónica (Fuster del Alcázar, 2003).

Por lo que respecta al Amor brujo, Manuel de Falla explica en su carta a Martínez Sierra que éste tenía bastante texto cuando se estrenó y que, más adelante al convertirlo en ballet, quedó reducido al de las canciones. También destaca el hecho de que él mismo realizó la escenografía de la nueva versión, así como la mise-en-scène, que, basada en su argumento, estableció para las representaciones del ballet.

Por otra parte, Falla reclama a Martínez Sierra un reparto más equitativo de los derechos de autor de la obra El sombrero de tres picos, aduciendo que, además de haber realizado un trabajo de ampliación de la partitura y de haberle añadido nuevas escenas y danzas, el ballet sólo tiene dos breves coplas como texto cantado.

Al final de la carta, Falla apela a sus circunstancias económicas que se agravan al no poder recibir ninguna aportación de España ni tampoco beneficiarse más que de una mínima parte de los derechos que producía su música.

La breve selección de la amplia correspondencia de Gregorio Martínez Sierra que hemos considerado en este artículo nos ha servido para ilustrar las relaciones que el dramaturgo mantuvo con los autores de su época y para, finalmente, contextualizar la producción literaria en una realidad social concreta.

\section{ANEXOS $^{2}$}

\section{Carta de Enrique Gómez Carrillo a Gregorio Martínez Sierra}

[Manuscrito membrete «Madrid Palace Hotel». Ca. 1920]

Mi querido Martínez Sierra, tenía yo la idea de que sí había sido la primera persona a quien había pedido el valioso apoyo de su colaboración para «Cosmópolis». ¿Está usted seguro de que no ha sido así? Entonces, la culpa es de mi costumbre de creer que he hecho lo que sólo he pensado hacer. Pero el corazón y etait.

¿Cuándo quiere V. almorzar o cenar con nosotros?

${ }^{2}$ En la transcripción de las cartas he realizado la acentuación, según criterios actuales. Asimismo, no he utilizado el subrayado original, que aparece en las mismas, que se ha sustituido por cursiva. 
Raquel $^{3}$, muy fiera va a buscarle a V. las fotografías en seguida.

Con mucho gusto autorizo a la colección miniatura o a cualquiera de V. para que publique mi Nuestra señora de los ojos verdes. Y muy pagado con encuentros con el gusto de verme en tan lindos tomitos.

Déme sus señas personales para ponerlas en la lista del servicio de Cosmópolis.

Un abrazo de su amigo y admirador

Gómez Carrillo

\section{Carta autógrafa de Juan Ignacio Luca de Tena a Gregorio Martínez Sierra}

San Sebastián

17-I-1938

Querido Gregorio: Al llegar a San Sebastián recibo su carta postal fechada el 1 de Enero en Jean-les-Pines. No ha llegado a mis manos la anterior de que me habla y nada sabía de [sic] que hubiese estado Vd. enfermo. Celebro la mejoría y le deseo su total y pronto restablecimiento.

Vengo del frente de Madrid, dentro de unos día iré a Sevilla y después al frente. Escríbame a San Sebastián, Miraconcha 4 y me llegarán más pronto sus cartas.

Trabajo con ilusión en una obra en verso cuya protagonista es una sirena. Las cosas superrealistas puede que sean - deben serlo - las preferidas del público de la post-guerra. Yo, desde luego, prefiero salirme de la realidad.

Un fuerte abrazo de su invariable amigo,

Juan Ignacio Luca de Tena

Señas de Sassone: Lima, no sé más

«de Nena Belmonte: Hotel Elyseé, 60 East 54th Street. New York.

3 La alusión al nombre de Raquel hace referencia a Raquel Meller, con quien Gómez Carrillo mantenía una relación en esa época. Véase Fuster del Alcázar (2006). 


\section{Carta de Juan Ignacio Luca de Tena a Gregorio Martínez Sierra}

[Mecanografiada. Membrete «Prensa Española. ABC-Blanco y Negro».]

San Sebastián, 17 de julio de 1938.

Sr. Don Gregorio Martínez Sierra.

Querido Gregorio: He tenido una gran satisfacción al recibir su carta con fecha 12 del corriente. Supongo en su poder una mía bastante larga que le escribí hace varios meses contestando otra suya.

No he entendido bien si van por fin o no a Buenos Aires; me figuro que la comedia mía a que se refiere Vd. es «Yo soy Brandel» segunda parte de «¿Quién soy yo?». Antes de comprometerme con Vd. para su estreno, desearía saber qué primer actor llevan Vds. Considero mucho más a propósito para Catalina otra obra mía de la que ya tengo dos actos; se titula «Espuma del mar», la protagonista es una sirena y uno de los actos, en verso, se desarrolla en el fondo del mar. La califico de «fábula escénica».

Mucho me agrada su coincidencia política conmigo. La guerra, como Vd. verá, va muy bien: estos días nuestros ejércitos avanzan rápidamente hacia Valencia y todo hace esperar que muy pronto podamos estar en Madrid.

Con mis saludos muy afectuosos para Catalina, le envía un fuerte abrazo, su buen amigo,

Juan Ignacio

\section{Carta de Manuel de Falla a Gregorio Martínez Sierra}

[Mecanografiada.]

Alta Gracia, 14 de septiembre 1943

Señor

Don Gregorio Martínez Sierra.

Querido Gregorio: Desde hace bastante tiempo, y especialmente desde que recibí la última liquidación de Chester, he querido escribirle, pero la falta de salud, primero, y luego una iritis que durante varios meses he venido padeciendo, me han impedido hacerlo. En dicha liquidación (demasiado 
modesta para ser la única que he recibido desde mucho antes de mi salida de España) hay a su favor una suma de $£ 34.3 .3$ que Chester le tiene reservada: Puede Vd. por lo tanto reclamársela pidiéndoles al mismo tiempo que le envíen directamente cuantos derechos le tengan igualmente reservados. Ya sabe Vd. la dirección postal: J. \& W. Chester Ltd., 11 Great Marlborough Street. London. W. 1. Desde hace años vengo reclamándoles, entre otras cosas, el pago de derechos que se han atribuido indebidamente en Estados Unidos, respondiendo siempre a mis reclamaciones que los autores españoles recibimos por mediación de la Sociedad de Madrid los derechos sinfónicos y el pequeño derecho, lo que, según afirma la Sociedad, no es cierto, por lo menos hasta mi salida de España. Para el futuro pretendo que la entidad Galaxi de New York me envíe directamente los derechos que por mis obras me correspondan, pero sin resultado hasta el momento, pues para hacerlo reclaman la autorización de Chester, y éste no la ha dado...

Ahora paso a otro asunto, también concerniente a nuestras obras. Quiero referirme al reparto de derechos por irradiación de las representaciones en el Colón y el que, sin duda por error involuntario, su representante le hizo reservar a Vd. un $20 \%$ en lugar del 15\%, que es el fijado por la Sociedad de Autores en sus repartos de derechos de irradiación. Así lo he hecho observar a Ricordi Americana (que, como Vd. sabe, administra con tanta eficacia como equidad, en cuanto a su comisión, nuestros derechos en el Colón) y así también espero de Vd. que lo confirme a dicha casa para que lo tenga en cuenta en las liquidaciones futuras, aunque dejando las pasadas tal como se han hecho.

Y a propósito del reparto de los otros derechos (los principales) por representaciones escénicas, voy a permitirme también rogar a usted que fije su atención y buena voluntad en la desproporción del porcentaje que se le reserva, tratándose de escenarios para ballets y no de libretos de ópera, en cuyo caso sí estaría dentro de las normas generalmente establecidas. Claro está que Vd. con razón, podrá decirme que así se ha venido haciendo desde que se estrenaron el Amor brujo y el Sombrero, pero si hace Vd. memoria recordará que el Amor brujo tenía cuando se estrenó bastante texto, el que luego, al convertirlo, de común y feliz acuerdo, en ballet, quedó reducido al de las canciones. Y por lo que concierne al escenario de la nueva versión, parte del mismo fue hecho por mí (como Vd. también recordará), así como la mise-enscène, que, basada en su argumento, debí establecer para las representaciones del ballet. Lo mismo digo respecto a los títulos de algunas de las escenas y danzas como, por ejemplo, el de la Ritual del Fuego, y, sobre todo, mi labor de ampliación de toda la partitura instrumental para ajustarla al plan or- 
questal de un ballet que pretendíamos (como se ha conseguido) fuese representado en grandes teatros.

En cuanto al Sombrero de tres picos, además de haber realizado este mismo trabajo de ampliación de la partitura y de haberle añadido nuevas escenas y danzas, como la del Molinero, el Corregidor, los Alguaciles, el Corregidor y la Molinera, la extensa Danza final, etc. etc., le ruego a Vd. recuerde que sólo tiene el ballet dos breves coplas como texto cantado, y aunque se ha hecho siempre el reparto de derechos en la misma forma que para el Amor brujo, ello ha obedecido a que Diaghilev, al hacerse con nosotros su contrato antes del estreno (autorizado por él) de la primera versión, fijó por error (uno de tantos demasiado frecuentes en él) para el reparto de la prima que nos abonó, el porcentaje que hubiese correspondido a los autores de una ópera. Yo no quise entonces poner las cosas en su punto exacto porque, como Vd. sabe, siempre he sentido gran antipatía ante toda posible discusión de ese carácter entre verdaderos amigos, y puede $\mathrm{Vd}$. creer que si ahora me permito hablarle de estas cosas, no sólo es pensando en los muchos años que ya lleva rigiendo esa norma de reparto, sino, muy especialmente, en razón a las tan especiales circunstancias económicas en que ahora me hallo, sin poder recibir nada de Espala ni tampoco beneficiarme más que de una mínima parte de los derechos que produce mi música. Aun del recurso de dirigir conciertos y de otras actividades propias de mi oficio, poco puedo servirme a causa del estado de mi salud. Claro está que, gracias a Dios (y no sé cómo dárselas), el buen ánimo nunca me falta, pero debo vivir en constante vigilancia para evitar deudas, que es una de las cosas que más me aterrorizan. En resolución (como diría Don Quijote): usted decide lo que crea justo y equitativo, y esa decisión la espero con toda confianza. Mientras, reciba el afectuoso saludo que le envío con el recuerdo de nuestra vieja amistad.

Manuel de Falla

Sale esta carta con gran retraso de su fecha a causa de una nueva recaída sufrida en mis males

S/c Chalet «Las Espinosillas»

Alta Gracia (C. de Córdoba) 


\section{REFERENCIAS BIBLIOGRÁFICAS}

CheCa Puerta, Julio (1998). Los teatros de Gregorio Martínez Sierra. Madrid: Fundación Universitaria Española.

FUSTER DEl AlCÁZAR, Enrique (2003). El mercader de ilusiones. La historia de Gregorio Martínez Sierra y Catalina Bárcena. Madrid: Ediciones y Publicaciones Autor.

- (2006). Archivo Gregorio Martínez Sierra-Catalina Bárcena.

MARTínez Sierra, María (2000). Gregorio y yo. Valencia: Pre-Textos.

O’Connor, Patricia (1987). Gregorio y María Martínez Sierra. Madrid: Editorial J. García Verdugo.

RAFAEL, Alfonso José (2001). «Verdades y mentiras en la vanguardia literaria venezolana». Cifra Nueva 13, 105-112.

Reyero, Carlos (1980). Gregorio Martínez Sierra y su teatro del arte. Madrid: Fundación Juan March.

SAntiago y Miras, M. Ángeles (2002). «Las colaboraciones de Gregorio Martínez Sierra en libretos zarzuelísticos». Espéculo 20, http://www.ucm.es/info/especulo/numero20/m_sierra.html. 